using twenty times as much medium as tissue, and changing as often as cloudiness appears. Small pieces of tumour are cut out of the mass ( $\frac{1}{8}$ to $\frac{1}{2}$ in. in diameter), and while yet warm are dropped into the medium at the temperature of the blood. Then the preparation is kept in running water until the colour is gone (circa 12 hours). It is then taken through the alcohols to bergamot oil (twice), xylol (twice), into bergamot again to prevent too violent evaporation of xylol in paraffin. When perfectly clear the sections are cut. The process will have taken about three days. The sections must not be thicker than $4 \mu$. If thicker the stain does not obtain fair play. Fixed on slide by alcohol method, and the paraffin being removed, the section is stained with 1 per cent. Grübler's eosin in alcohol, being left all night (alcohol removed by water) and counterstained with 1 per cent. methylene blue " $\mathrm{O}$ " in water and 10 per cent. alcohol. Wash out stain with absolute alcohol, drop into bergamot oil free of alcohol through xylol to gum--thus in xylol (or failing this Canada balsam in xylol).

The tissue is pale red with bluish nuclei, the amœbæ intensely red with deep blue nuclei.

"Cancriamoba macroglossa," 7 to $30 \mu$, extrudes a tongue-like process and enters epithelial cells, devouring the contents and eventually lying in the vacuole thus caused. The pseudopodium acts as a locomotive organ and as a suctorial one. Several cells may be attacked at the same time by different pseudopodia of the same amœba. Sporulation probably occurs by division of the nucleus. Plimmer's organisms are probably the spores. Amitotic division also occurs.

He describes the epithelial proliferation as an attempt to enclose the parasites by means of the cells, which are best able to deal with them. Cell-nests are produced by the enclosure of the parasites, which are found in the centre, sometimes endeavouring to pierce the invading wall, sometimes dead and solidified. The presence of pseudopodia have been found actually invading cells, and the fact that the amœbæ sporulate exclude the possibility that they are blastomycetes or other fungi.

The sensitiveness of the parasites to cold suggests the treatment of carcinomata by freezing.

Statistics. - Mahler ${ }^{11}$ publishes the impressions of 161 cases of mammary carcinomata. As to ætiology, he finds no positive support in the influence of the menopause, number of births, lactation, and trauma; but insterstitial mastitis he considers to play an rtiological part.

Most of the tumours were mixed forms of scirrhus and medullary cancer : 16.4 per cent. of the cases presented no swelling of glands.
The axillary glands were affected 127 times

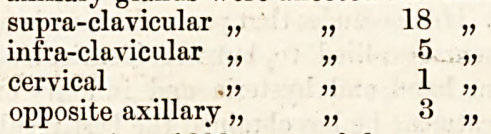

At the operations since 1890, most of the most superficial layer of the Pectoralis major was removed; but never the whole breast muscles.

Of 133 cases operated on-

21 per cent. remain well after 3 years
$14 \cdot 1 \quad " \quad " \quad$ " $"$ are dead."

Seventy-one recurrences were met with, and 30 metastases in inner organs.

Treatment.-Petrini-Galatz, ${ }^{12}$ of Bucharetz, communicates the case of a man of 40 years, who suffered from multiple tumours, occupying the fauces, the palatine arch, the gums, the forthead, the neck, \&c. Each of these tumours was of the size of a small chestnut, and presented a semi-hard consistency. They were situated under the skin, to which they were partly adherent, or infiltrated the deep tissues considerably, in the buccal cavity for instance. Histological examination of a portion cut out showed that it consisted of an embryonic vascular sarcoma. The patient was treated with "cacodylate of soda," given in intramuscular injections of about 0.05 centigr., then 0.08 , $0 \cdot 1,0 \cdot 12$ centigr. per diem. These injections were administered partly into the buttocks, partly into the tumours of the subhyoid region; after fifty injections the resolution of the tumours was almost completely produced, and the patient enjoyed very good health. The result seems to be that non-melanotic multiple sarcomata, if treated early, are amenable to treatment with arsenic by injection.

Cases.-A case of epithelial cancer of bone following a fracture is reported from Maier's clinic. ${ }^{13}$ It occurred in a girl aged 20, who in 1896 injured her ulna, the injury causing considerable pain at the time. In 1897 a thickening was noticed, and a skiagram showed a separation of the epiphysis, with a clear intervening space. Diagnosis was made of mytloid sarcoina. The ulna was resected and ivory implanted; three years afterwards the union was found strong. The preparation showed in the centre of the bone a typical epithelial cancer with marked cornification. The writer explained the case by supposing that the injury carried a deep process of epithelium downwards between the portions of fractured bone.

Newbolt ${ }^{14}$ reports the case of a woman who had a duct carcinoma in the left breast coincidently with a glandular carcinoma in the right.

Rolleston ${ }^{15}$ brings forward a case of primary carcinoma of the vermiform appendix.

"Med. Chron., June, 1900. '7 Med. Chron., June, 1900. ' ${ }^{8}$ Brit. Med. Jour., Mar. 31, 1900. Amer. Jour. Med. Sci., Aug. 1900. '10 Med. Rec., July 7, 19c0. " Cent. für Chir., June 23, 1900. "2 La Sem. Méd., Aug. 15, 1900. ${ }_{13}$ Cent. für Chir., June 23, 1900. ${ }^{14}$ Lancet, May 26, 1900. '13. Lancet July $7,1903$.

\section{Progress in Pediatrics.}

Day Terrors (Pavor Diurnus).-This affection is to be distinguished from night terrors in that the latter occur while the patient is asleep, and the former while he is awake. It is a rare condition, of which Henoch has recorded two cases, and G. F. Still ${ }^{14}$ has recently published three more. The symptoms are fairly constant. A nerrous, excitable child, in the midst of his ordinary occupations, will suddenly and without any apparent cause begin to scream. He looks terrified, rushes to his mother, says some person is coming after him, or that he hears something alarming, and cannot be easily pacified. There is no loss of consciousness. The attacks may vary in duration from a few seconds to a quarter of an hour, and in frequency from one in a fortnight up to twenty in a day. They may or may not be associated with night terrors. The fact that the two conditions are associated in some cases points to a similarity in causation, and disproves the common assumption that sleep is a necessary factor in the production of night terrors. Dr. Still discusses the relation of these terrors to epilepsy, and does not find 
that the subjects of them are specially liable to that affection. He concludes that pavor diurnus is a paroxysmal psycho-neurosis allied to, but not identical with, epilepsy on the one hand and hysteria and insanity on the other. In its treatment he has obtained the best results by curing the condition of chronic intestinal catarrh which is usually present as the result of bad feeding. For this purpose simple plain focd and the use of citrate of potash with compound decction of aloes will ke found useful. Sedatives in the form of bromides and belladonna will ke required for a time. The nerrous character of the child must also be recognised, and both work and play regulated so as to avoid mental strain and excitement.

Measles complicated with Meningitis and Spinal Myelitis. - A very interesting case of this nature, terminating in recovery, is recorded by E. F. Eliot. ${ }^{15}$ The patient was a girl of eighteen years, healthy up to the onset of measles. On the fourth day after the eruption epileptiform seizures cccurred, and were followed later by signs of spinal irritaticn. There was incontinence of urine and fæces, the lower limbs were paralysed, the upper limbs were paretic, and the knee jerks and plantar reflexes were abolished. The condition for some time was apparently hopeless, but after a prolonged illness complete recovery eventually took place.

my otonia Congenita (Thomsen's Disease). - A case of this rare affection was shown by Leonard Guthrie ${ }^{16}$ at a meeting of the Medical Society of London. The patient was a boy ten years old, who had been brought to the hospital because of the awkwardness of his movements. Transient spasticity affected both the lower and the upper extremities. He took hold of anything with difficulty, and could not let go easily. Sometimes he had to open out the fingers of one hand with the other before being able to grasp an object. He had a difficulty in feeding himself at times. His gait when starting was jerky, and his progression up a stair was laboured. The condition of spasm was much more marked after rest, and was lessened after exercise or when his attention was distracted from himself. The spasm persisted during sleep. Although the symptoms pointed clearly to Thomsen's disease, there was no family history of this affection in this case.

14
1900.

\section{NEW APPLIANCES AND THINGS MEDICAL.}

\section{[We shall be glad to receive, at our Office, $28 \& 29$ Southampton Street, Strand, London, W.C., from the manufacturers, specimens of all new preparations} and appliances which may be brought out from time to time.]

\section{HEMOGALLOL AND STYPTICIN.}

(E. Merck, 16 Jewry, London, E.C.)

Hæmogallol is an organic iron preparation obtained by a process invented by Professor Kobert, which comprises the reduction of hæmoglobin by such bodies as zinc dust and pyrogallol. The resulting body contains a large percentage of iron, and fulfils the conditions which are now demanded of iron preparations if they are directly to assist in the formation of hæmoglobin in the body. These conditions may be generally stated as follows:-The iron must be absorbed from the alimentary tract; it must remain in the system for an appreciable period-for instance a few days; and part of it must be excreted"by the kidneys in combination with uric acid. An iron preparation fulfilling these conditions may be assumed to have entered into close combination with the iron containing proteids of the body, and hence in a large measure to have corrected the conditions which are responsible for the anæmic state. Three or four 5 -grain tabloids of hæmogallic may be regarded as one of the best medicinal treatments of anæmia. Stypticin is an oxidation product of narcotin, an alkaloid of opium; in its therapeutic and chemical properties it resembles hydrastinine, and is useful in the same conditions for which the latter drug is prescribed; that is to say, for menorrhagia and other uterine disorders. It is a styptic with anodyne properties. It should be given in 1-grain doses three or four times a day.

\section{THE ALLENBURYS SERIES OF INFANT FOODS.}

(Allen and Hanburys, Limited, Plough Court, Lombard

Street, E.C., and Bethnal Green, London, E.)

Although we are well acquainted with the Allenburys method of infant feeding, we have recently examined more carefully into the details on which depend the success or failure of the practical application. We ourselves have occasionally robserved failures, and we have heard of others, and in each case we have come to the conclusion that the directions supplied with the foods have not been implicitly followed. But at the same time we cannot altogether absolve the manufacturers from blame. Their directions are not sufficiently precise for so delicate a matter as infant feeding. For instance, a child one day old should not be given the same quantity as a child one week old; if this information is to be gathered from the directions, it is by implication and not directly. The system is well conceived, and if carried out exactly and scientifically should certainly, and can, as we are able personally to testify, give most excellent results. The objects which are fulfilled by the method are manifold. In the first place the basis of the food is milk in a specially prepared and modified form-when made according to the directions it approximates to the chemical constitution of maternal milk. It can be digested far more easily than ordinary forms of diluted and modified milk, and is especially suitable for delicate and premature infants. Secondly, the three varieties of food are appropriately modified to the digestive powers of the infant during the successive periods of its life. The milk food No. 1 is intended for the first three months of life, and is prepared for use by mixing with water only. It is the only food of the kind with which we are acquainted which contains a sufficiently high percentage of fat for the requirements of infants at this age. If the amount of sugar in the food as prepared for use.could be reduced from 10 per cent. to 6 per cent., without interfering with the percentage of the fat, the food would be practically perfect, if supplemented, as the manufacturers advise, or rather insist, with some antiscorbutic element, such as meat or grape juices. The milk food No. 2 is similar to food No. 1 , but contains in addition maltose, dextrine, soluble phosphates, and albuminoids. When mixed according to the directions it again approximates to the constitution of maternal milk, and affords an easy stepping-stone to the third stage of the series. This food No. 3 is a partially digested farinaceous food, which should be added to diluted milk. This latter food, though qualitatively excellent, should be given in measured and exact quantities. Owing to the ease with which carbohydrates can be given in excess during this stage (i.e., six months to a year), we are again of opinion that the directions are not sufficiently precise. Taken as a whole, the Allenbury method is an excellent one: it provides variety in well-regulated gradation in accordance with the infant power of digestion. 'The possibility of infection by means of contaminated milk is impossible-at any rate during the first six months-and scurvy rickets should not ensue if occasionally fresh food as directed is made a part of the régime. 